\title{
CUERPO EDITORIAL
}

\section{DIRECTOR}

- Dr. Esteban Sanchez Gaitan, Dirección regional Huetar Atlántica, Limón, Costa Rica.

\section{CONSEJO EDITORIAL}

- Dr. Cesar Vallejos Pasache, Hospital III lquitos, Loreto, Perú.

- Dra. Anais López, Hospital Nacional Edgardo Rebagliati Martins, Lima, Perú.

- Dra. Ingrid Ballesteros Ordoñez, Pontificia Universidad Javeriana, Bogotá, Colombia.

- Dra. Mariela Burga, Hospital Nacional Edgardo Rebagliati Martins. Lima, Perú.

- Dra. Patricia Santos Carlín, Ministerio de Salud (MINSA). Lima, Perú.

- Dr. Raydel Pérez Castillo, Centro Provincial de Medicina Deportiva Las Tunas, Cuba.

\section{COMITÉ CIENTÍFICO}

- Dr. Zulema Berrios Fuentes, Ministerio de Salud (MINSA), Lima, Perú.

- Dr. Gerardo Francisco Javier Rivera Silva, Universidad de Monterrey, Nuevo León, México.

- Dr. Gilberto Malpartida Toribio, Hospital de la Solidaridad, Lima, Perú.

- Dra. Marcela Fernández Brenes, Caja costarricense del Seguro Social, Limón, Costa Rica

- Dr. Hans Reyes Garay, Eastern Maine Medical Center, Maine, United States.

- Dr. Steven Acevedo Naranjo, Saint- Luc Hospital, Quebec, Canadá.

- Dr. Luis Osvaldo Farington Reyes, Hospital regional universitario Jose Maria Cabral y Baez, Republica Dominicana.

- Dra.Caridad Maria Tamayo Reus, Hospital Pediátrico Sur Antonio María Béguez César de Santiago de Cuba, Cuba.

- Dr. Luis Malpartida Toribio, Hospital Nacional Daniel Alcides Carrión, Callao, Perú.

- Dra. Allison Viviana Segura Cotrino, Médico Jurídico en Prestadora de Salud, Colombia.

- Mg.Luis Eduardo Traviezo Valles, Universidad Centroccidental "Lisandro Alvarado" (UCLA), Barquisimeto, Venezuela.

- Dr.Pablo Paúl Ulloa Ochoa, Instituto Oncológico Nacional "Dr. Juan Tanca Marengo", Guayaquil, Ecuador.

\section{EQUÍPO TÉCNICO}

- Msc. Meylin Yamile Fernández Reyes, Universidad de Valencia, España.

- Lic. Margarita Ampudia Matos, Hospital de Emergencias Grau, Lima, Perú.

- Ing. Jorge Malpartida Toribio, Telefónica del Perú, Lima, Perú.

- Srta. Maricielo Ampudia Gutiérrez, George Mason University, Virginia, Estados Unidos.

\section{EDITORIAL ESCULAPIO}

50 metros norte de UCIMED, Sabana Sur, San José-Costa Rica Teléfono: 8668002

E-mail: revistamedicasinergia@gmail.com

\section{ENTIDAD EDITORA}

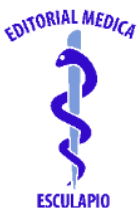

Teléfono: 8668002
SOCIEDAD DE MEDICOS DE AMERICA

Frente de la parada de buses Guácimo, Limón. Costa Rica

Sociedaddemedicosdeamerica@hotmail.com

https://somea.businesscatalyst.com/informacion.html 


\title{
Actualización en dermatitis atópica: líneas de tratamiento
}

\author{
Update in atopic dermatitis: treatment lines
}

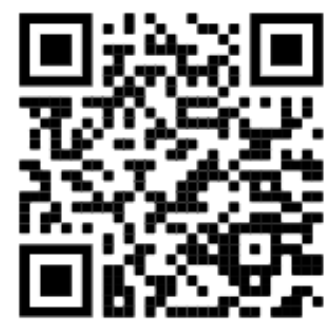

${ }^{1}$ Médica general, graduada de la Universidad Latina de Costa Rica (U.Latina), cod. MED15602. dralopez15602@gmail.com

2 Médica general, graduada

de la Universidad de Ciencias Medicas (UCIMED), cod. MED15313. eka91@me.com

${ }^{3}$ Médica general, graduada de la Universidad de Ciencias Medicas (UCIMED), cod.MED15236. genesisc10@gmail.com
${ }^{1}$ Dra. Silvia López Vargas Investigadora independiente, San José, Costa Rica (D) https://orcid.org/0000-0001-9103-7180

${ }^{2}$ Dra. Erika Núñez Picado Investigadora independiente, San José, Costa Rica (iD) https://orcid.org/0000-0002-3797-1236

${ }^{3}$ Dra. Génesis Chaverri Padilla Investigadora independiente, San José, Costa Rica (1D) https://orcid.org/0000-0002-4454-3969

RECIBIDO

01/05/2020

CORREGIDO

ACEPTADO

08/07/2020

$20 / 07 / 2020$

\section{RESUMEN}

La dermatitis atópica es la dermatosis inflamatoria más frecuente, con un pico de prevalencia en la niñez temprana y con mayor porcentaje de afectación en países industrializados. Entre las principales características de este trastorno crónico y/o recidivante se destacan, el prurito, la dermatitis eccematosa, el compromiso facial y de regiones extensoras en la niñez temprana, mientras que el eccema y liquenificación en pliegues es más usual en niños y adultos. En los últimos años ha habido un avance en el conocimiento de la patogenia, centrada en la vía Th2, pero con una participación significativa de la vía Th22 y de los ejes Th1 y Th17, de la disfunción de la barrera epidérmica, el prurito y la señalización JAK/STAT, este progreso ha condicionado innovaciones en los tratamientos tópicos y sistémicos.

PALABRAS CLAVE: alérgenos; dermatitis; eccema; prurito.

\section{ABSTRACT}

Atopic dermatitis is the most frequent inflammatory dermatosis, with a peak of prevalence in early childhood, the evidence highlights a higher percentage of involvement in industrialized countries. Among the main characteristics of this chronic and / or recurrent disorder, pruritus, eczematous dermatitis, facial involvement and extensor regions in early 
childhood stand out, while eczema and lichenification in folds is more common in children and adults. In recent years, there has been an advance in the knowledge of pathogenesis, focused on the Th2 pathway, but with a significant participation of the Th22 pathway and the Th1 and Th17 axes, of dysfunction of the epidermal barrier, pruritus and JAK / STAT signaling, this progress has conditioned innovations in topical and systemic treatments.

KEYWORDS: allergens; dermatitis; eczema; pruritus.

\section{INTRODUCCIÓN}

La dermatitis atópica es una es una entidad que se manifiesta con más frecuencia en los primeros años de vida, sin embargo en algún porcentaje de la población persiste en la edad adulta. Se caracteriza por ser una dermatosis crónica, multifactorial, altamente pruriginosa que cursa con episodios de crisis y remisiones, se divide en fases según sus características clínicas y la edad de presentación, su diagnóstico se establece mediante una adecuada anamnesis y examen físico del paciente, existen diversos diagnósticos diferenciales y múltiples líneas de tratamiento según la severidad y la etapa de presentación de la enfermedad.

Al ser una patología que se presenta con frecuencia en la consulta médica es importante reconocerla, para poder brindar un abordaje integral al paciente y buscar la opción terapéutica más adecuada para su condición clínica.

Actualmente los estudios revelan que el sistema inmunológico tiene un papel importante en su patogénesis, y los nuevos tratamientos para pacientes con poca respuesta a las terapias convencionales se enfocan en el bloqueo de estas vías de respuesta magnificada. El objetivo de esta revisión bibliográfica es la recopilación de información actualizada sobre los avances en dermatitis atópica a nivel mundial y evidenciar como en los últimos años de estudio se aportan nuevas líneas para su tratamiento, de esta forma se brinda una guía actualizada de abordaje terapéutico en los diferentes estadios de la patología al personal de salud. Dentro de las nuevas opciones terapéuticas destacan los fármacos de acción biológica, para el control de pacientes con poca respuesta a las terapias de primera línea o convencionales.

\section{MÉTODO}

Se realizó una revisión bibliográfica de artículos que se comprenden del año 2017 al año 2020, utilizando el buscador Pubmed y las palabas "atopic dermatitis, atopic dermatitis review, atopic dermatitis guidelines, atopic dermatitis treatment", también se utilizó el libro de texto Dermatología en medicina general, edición 9, versión en inglés. Se analizaron 45 artículos, de los cuales se seleccionaron 25 artículos para la creación de esta revisión, la selección se basó en el cumplimiento de los criterios de interés como: fecha de publicación menor a tres años, inclusión de nuevas lineas terapéuticas, explicación detallada de los criterios para el diagnóstico y la utilización de escalas validadas internacionalmente. 


\section{DEFINICIÓN}

La dermatitis atópica es una enfermedad prevalente a nivel mundial que predomina en la edad pediátrica, sin embargo un porcentaje de la población la padece en la adultez. Se manifiesta como una patología crónica e inflamatoria que presenta brotes y remisiones, sumamente pruriginosa y en la que el paciente sufre una afectación importante en la calidad de vida (1-4). Sus manifestaciones fenotípicas son variadas, influyen diversos factores como el medio ambiente en el que se desarrolle el paciente, su respuesta inmunológica y la genética (3).

\section{EPIDEMIOLOGÍA}

Se ha establecido la dermatitis atópica como la enfermedad inflamatoria de la piel más frecuente. Datos obtenidos en la región occidental muestran que en la población pediátrica se presenta hasta en un $15 \%$, siendo más prevalente en niñas que en niños y con un rango de edad de presentación entre el primer y quinto año de vida, mientras que en adultos se estima en un $10 \%$ del total la población $(1,3)$. En los últimos años se ha evidenciado que esta patología es más frecuente en países industrializados, siendo hasta tres veces más diagnosticada que en países subdesarrollados. La enfermedad no se asocia a mortalidad, sin embargo tiene implicaciones importantes en la calidad de vida del paciente y su entorno laboral, social y económico (5). Aproximadamente un $50 \%$ de los pacientes afectados por dermatitis atópica desarrolla asma y alergias nasales como la rinitis, sin embargo las características de cada paciente juegan un papel importante en esta
estadística.(6)

\section{ETIOLOGÍA}

La disfunción de la barrera cutánea es el evento primario en el desarrollo de la enfermedad y están implicados en la pérdida de la función de la barrera epidérmica, la regulación genética negativa de la envoltura queratinizada, los niveles disminuidos de ceramida, la pérdida de agua a través de la epidermis $y$ el incremento de enzimas proteolíticas endógenas (3,7-9). Sin embargo el factor predisponente más relevante, es la mutación que conlleva a la pérdida de función de la proteína de la barrera epidérmica, el gen de la filagrina, localizado en el cromosoma 1q21 (9-11).

\section{PATOGENIA}

El pilar en la evolución de la DA es la alteración de la inmunidad, se ha considerado que existe un desbalance entre las respuestas de los linfocitos $T$ helper Th1/Th2, con predominio de la respuesta Th2, a su vez, la mayoría de estos pacientes tienen incremento en el número de eosinófilos circulantes y en los niveles de lgE $(3,9)$.

Los linfocitos Th2 producen interleucina IL-4 e IL-13 encargadas de inhibir la expresión de la filagrina, mediar la síntesis de $\lg$ y estimular tanto la diferenciación a nivel de los eosinófilos como la expresión de las moléculas de adhesión en las células endoteliales, todo esto permite establecer una relación entre la disfunción inmunológica y la alteración de la función de la barrera características de la DA. En relación a lo mencionado anteriormente, se ha 
estudiado el papel de la vía JAK-STAT (Janus kinase/signal transducer and activator of transcription) activada por la IL-4 en la desrregulación inmune de la DA y su participación en la respuesta Th2 (3,8-11). En años recientes, diversos estudios han centrado la patogenia de la DA en una respuesta Th2/Th22 durante todo su curso, con cierta participación de Th17 y con una contribución adicional del eje Th1 en su etapa crónica $(3,8,9,11)$.

Existen otras piezas fundamentales en el proceso patogénico, los queratinocitos, estos producen citocinas como la linfopoyetina estromal tímica (TSLP) y la IL-33, que inducen la expresión de OX40L y por ende, amplifican la respuesta Th2 $(3,9,11,12)$.

En la epidermis de los pacientes con DA se encuentran incrementadas las células de Langherans y las células dendríticas inflamatorias epidérmicas, ambas con afinidad para los receptores para la $\lg E$ (FceRI). Las células dendríticas detectan alérgenos y patógenos del ambiente mediante el reconocimiento del patrón de los receptores tipo Toll (TLR) produciendo una alteración en el $\mathrm{pH}$ de la piel y una disminución en la actividad de los péptidos antimicrobianos, situación que favorece la colonización por Staphylococcus aureus y que perpetúa el estado de inflamación crónica $(3,9,10,13)$.

Con respecto al prurito, se han relacionado las interleucinas IL-4 e IL-13 involucradas en la síntesis de IL-31 e IL5 , las cuales facilitan el reclutamiento de Th2 y de eosinófilos. A su vez, el rascado acentúa la disfunción de la barrera cutánea y permite la colonización por Staphylococcus aureus, minimizando la expresión de péptidos antimicrobianos $(3,9)$.

\section{MANIFESTACIONES CLÍNICAS}

Se presenta como un brote donde la lesión elemental es la vesícula, asociada a xerodermia y signos de rascado. Pueden encontrarse placas eritematosas conformadas por vesículas y pústulas que posteriormente se liquenifican por el rascado secundario al prurito intenso que caracteriza a esta patología. Es una entidad que presenta un curso intermitente entre períodos de brotes y remisiones (2). La dermatitis atópica puede estratificarse en aguda, subaguda y crónica según su tiempo de evolución y la edad de presentación:

- La fase aguda: se manifiesta con más frecuencia durante la infancia temprana o fase del lactante se observan lesiones papulovesiculares asociadas a prurito intenso, generalmente exudativas o húmedas que se forman sobre una base de eritema y asocian aumento del calor local. Al ser lesiones húmedas posteriormente podrían aparecer costras predominantemente melicéricas. Las lesiones usualmente se localizan en superficies de extensión como codos y rodillas, también se ubican en las mejillas y cuero cabelludo. El área del pañal no se encuentra comprometida en estos casos.

- La fase subaguda: se caracteriza por la formación de placas vesiculopapulares con eritema leve, sobreelevadas por el engrosamiento cutáneo, presentan descamación blanquecina asociada a estigmas de rascado. Las zonas de flexión suelen ser las más afectadas, entre estas la 
fosa poplítea y antecubital, así como palmas, tobillos y cuello. Esta fase se observa comúnmente durante la infancia tardía, una variante de presentación en esta etapa es el eczema numular, el cual tiene forma redondeada o de moneda, como su nombre lo indica, conformado por pápulas altamente pruriginosas sobre una base eritematosa.

- La fase crónica se desarrolla usualmente durante la adolescencia y en la edad adulta, aparecen placas crónicas liquenificadas, asociadas a un aumento visible de las líneas en la piel que pueden progresar a excoriaciones y posteriormente fibrosis, con frecuencia se localizan en el dorso de manos y pies, zonas de flexión, parpados y pliegues $(2,5,14)$. Otros signos de dermatitis atópica severa son: eritema facial, pérdida lateral de las cejas, pliegue de Dennie-Morgan, poiquilodermia y el signo de cuello reticulado o sucio.

\section{DIAGNÓSTICO}

En la dermatitis atópica la clave del diagnóstico se basa en una adecuada anamnesis y las manifestaciones clínicas del paciente. En muy pocos casos se necesitan estudios complementarios 0 una biopsia cutánea. Cuando estos son necesarios se utilizan para descartar otros posibles diagnósticos 0 en pacientes que no responden adecuadamente a las diversas líneas de tratamiento.

Actualmente el diagnóstico de la dermatitis atópica es complejo, debido a que no existe un consenso estandarizado. En 1980 se crearon los criterios de Hanifin y Rajka considerados el "Gold standard", los cuales han sido modificados en los últimos años en diversos países. Los criterios de Hanifin y Rajka originales constan de 4 criterios mayores y 23 menores y el diagnostico se establece con la presencia de 3 o más criterios mayores y 3 o más criterios menores $(1,2,5)$ :

- Criterios mayores: prurito, morfología y distribución típica (liquenificación en zonas de flexión en adultos, compromiso facial y de superficies extensoras en niños), dermatitis crónica, historia familiar o personal de atopia.

- Criterios menores: cataratas, queilitis, conjuntivitis recurrente, eczema, eritema o palidez facial, alergias alimentarias, dermatitis no alérgica en las manos, elevación de IgE con reactividad cutánea de tipo I, pliegue de Dennie-Morgan, prurito al sudar, queratosis pilar, queratocono, dermatitis del pezón, hiperpigmentación periorbitaria (ojeras), hiperlinearidad palmar, pitiriasis alba, dermografismo, intolerancia a la lana, xerosis.

Se han creado escalas que determinan el grado de afectación en la calidad de vida, entre ellas el EASI y el SCORAD (1). La más validada es el SCORAD que evalúa tres aspectos:

1. Extensión, porcentaje de superficie corporal comprometida, donde se utiliza la regla de los 9 , misma escala utilizada en quemaduras.

2. Clínica y gravedad de las lesiones.

3. Síntomas subjetivos, utilizando una escala visual del cero al diez.

Una vez valorados los tres aspectos del SCORAD se establece el grado de 
afectación al paciente en leve, moderado y severo $(1,4,6,15)$.

La escala EASI estima el grado de afectación mediante el porcentaje de área corporal comprometida que se divide en 4 regiones y la severidad de las lesiones, no evalúa los síntomas subjetivos del paciente $(1,5)$.

Dentro de los diagnosticos diferenciales se encuentran: $(6,10,14,16,17)$. Dermatitis por contacto, reacciones alérgicas a fármacos $\mathrm{y} / \mathrm{o}$ alimentos, infecciones por hongos (tiña capitis, tiña corporis), escabiosis, dermatitis seborreica, psoriasis, deficiencias nutricionales, enfermedades del colágeno, queratosis pilaris, deficiencias inmunitarias, linfoma cutáneo de células $\mathrm{T}$, dermatosis fotosensibles, prurigo simple.

\section{COMPLICACIONES}

La relación entre DA y alergias alimentarias, asma, rinitis alérgica, como parte de la marcha atópica, ha sido extensamente documentada.

Se asocia también con un riesgo aumentado de dermatitis de contacto alérgica, dermatitis de las manos y dermatitis de contacto irritativa $(6,9,10,11,16)$. Existe un aumento de la susceptibilidad a infecciones cutáneas bacterianas y virales diseminadas, así como a infecciones extracutáneas. Con respecto a las infecciones virales, la más severa es el herpes simple, una diseminación cutánea del tipo 1 , que afecta a todas las edades, se caracteriza por una erupción variceliforme de Kaposi en donde aparecen vesículo-pústulas umbilicadas,confluentes con presencia de zonas hemorrágicas y costras $(1,6,14,17,18)$. En más del $90 \%$ de las lesiones de la piel producidas por DA se encuentra S.aureus, se sospecha ante la presencia de foliculitis, pioderma 0 costras melicéricas. Las infecciones por $\mathrm{S}$. aureus de localización profunda son raras y se debe orientar a un síndrome de inmunodeficiecia como el Síndrome hiper-lgE $(10,13)$.

El prurito y el dolor son los síntomas más comúnmente reportados, tanto en niños como en adultos, provocan efectos perjudiciales en la calidad de vida. La incapacidad para conciliar el sueño y los despertares frecuentes disminuyen la calidad del mismo, implicando un bajo rendimiento escolar, trastornos por déficit de atención e hiperactividad y mayores riesgos de accidentes. Se ha relacionado también con condiciones neuropsiquiátricas como trastornos por depresión y ansiedad $(11,14,19,20)$.

Asimismo se ha asociado a comorbilidades, tales como, la obesidad, síndrome metabólico, enfermedades cardiovasculares, enfermedades autoinmunes y desórdenes gastrointestinales como síndrome de intestino irritable y gastritis $(6,11)$.

\section{TRATAMIENTO}

La selección del tratamiento se basa en la gravedad de la enfermedad, con ajustes según la edad del paciente, comorbilidades asociadas o no con atopia, respuesta y adherencia al tratamiento y costo $(1,2,4,6)$.

\section{- Hidratación}

La terapia más importante en todos los niveles de severidad es la hidratación de la piel mediante el uso continuo de humectantes, su objetivo es restaurar la barrera cutánea, ya 
que reduce la sequedad dérmica (xerosis) y el prurito asociado, así como, el número de brotes y la necesidad de preparaciones tópicas de esteroides. Inclusive se plantea la hidratación cutánea temprana (primeros seis meses de vida) como un método eficaz de prevención $(14,20,21$,$) .$

La hora del baño debe ser breve, no superior a los 5 minutos y a una temperatura tibia, las temperaturas altas generan mayor vasodilatación lo que favorece el prurito. Estos pacientes deben secarse sin producir fricción en la piel y aplicar los emolientes a los tres minutos de finalizado el baño ya que es el momento de mayor absorción. La elección del humectante se basa en factores como; el sitio de aplicación, la preferencia del paciente y el grado de sequedad de la piel, existen una variedad de opciones de humectantes que incluyen cremas, ungüentos, lociones y geles. $(1,2,4,6,11,14,22$,

\section{- Identificación de factores desencadenantes}

La identificación de factores desencadenantes individuales es crucial para el tratamiento de la DA y su erradiación permite fases más largas de remisión. Numerosos factores pueden irritar la piel y provocar eccemas, tales como el contacto con sustancias químicas y materiales sintéticos, exposición a temperaturas extremas $y$ contaminantes del aire. Se recomienda el uso de jabones con $\mathrm{pH}$ neutro y productos de cuidado personal libres de alcohol o perfume, lavado previo de la ropa nueva, prefencia por prendas claras y de algodón, así como utilizar detergentes líquidos y no en polvo y evitar actividades deportivas que impliquen una sudoración excesiva $(1,6,10,20,23)$.

Mediante un minucioso interrogatorio y pruebas cutáneas selectivas 0 cuantificación de los niveles séricos de $\lg E$, se pueden identificar $y$ controlar potenciales alérgenos, tales como, alimentos, alérgenos del aire como ácaros del polvo, caspa de animales, levaduras, pólenes, temperaturas extremas, sudoración excesiva $(23,24)$.

\section{- Corticosteroides tópicos}

Los corticosteroides tópicos se consideran la base del tratamiento antiinflamatorio de las lesiones eccematosas, sin embargo, debido a sus efectos adversos se prefiere su uso solamente ante las exacerbaciones agudas, se clasifican según su potencia, en siete clases.

La potencia esta directamente relacionada con el grosor de la piel, se debe evitar el uso de corticosteroides potentes fluorados en la cara, áreas de los genitales y en los pliegues, para estas áreas se recomiendan los de baja potencia. Los de potencia intermedia se pueden utilizar durante lapsos más prolongados, con afectación de zonas como el troco y las extremidades. Los de potencia ultraalta en zonas de liquenificación, durante períodos muy cortos y los de potencia máxima deben limitarse a lesiones refractarias. Entre los efectos adversos se han evidenciado la 
atrofia cutánea, el desarrollo de estrías, acné rosácea, dermatitis perioral. En los casos de liquenificación extrema el uso de vendajes húmedos con los esteroides tópicos durante períodos cortos es recomendable y seguro $(1,2,5,6,10)$.

- Inhibidores tópicos de la calcineurina

Los inhibidores tópicos de la calcineurina, tales como el tacrolimús y el pimecrolimús, son el tratamiento de segunda línea, aprobados para el tratamiento de la DA moderada a severa en adultos y niños mayores de dos años.

Son de alta utilidad en áreas de piel sensible, como el rostro, genitales y en los pliegues, no se han asociado con atrofia de la piel. La sensación transitoria de quemazón cutánea se destaca como efecto adverso $(6,11,17)$.

\section{- Antihistamínicos}

Debido a que la histamina es apenas uno de los múltiples mediadores que producen el prurito, ciertos pacientes obtienen tan solo un mínimo beneficio de la terapia con antihistamínicos.

Los de primera generación se utilizan como coadyuvantes por su efecto sedante, sin embargo a pesar de promover el sueño, reducen el sueño de movimientos oculares rápidos (REM), afectando la calidad del mismo. Para mitigar la sensación de prurito se pueden realizar baños con hipoclorito de sodio ya que disminuyen la carga bacteriana $(6,10,14,20)$.

\section{- Antibióticos}

Los antibióticos no se recomiendan de forma rutinaria, solamente si existe sobreinfección bacteriana, la terapia antiobiótica antiestafilocócica es altamente útil en pacientes intensamente colonizados con S. aureus. Si se sospecha infección por el virus herpes simple, se debe suspender de manera temporal el uso de agentes tópicos antiinflamatorios e iniciar terapia vía oral con Aciclovir $(10,13,17)$.

\section{- Terapia sistémica}

La decisión de iniciar la terapia sistémica debe basarse en la severidad de los síntomas, en la calidad de vida del paciente, así como en el riesgo/beneficio, de manera individualizada. La administración de corticosteroides sistémicos debe realizarse durante un corto período, es necesario un descenso gradual e intensificar el cuidado de la piel para prevenir las exacerbaciones de DA de rebote (6, 20).

\section{- Ciclosporina}

La administración de ciclosporina esta aprobada en la DA severa, se evidencia una reducción de la enfermedad cutánea y mejoría en la calidad de vida del paciente, aproximadamente la mitad de los pacientes mejoran después de 6-8 semanas de tratamiento, sin embargon entre sus efectos colaterales se exponen la hipertensión arterial y el deterioro de la función renal $(6,17,20)$. 


\section{- Antimetabolitos}

Respecto al tratamiento con antimetabolitos, se puede considerar la administración de micofenolato de mofetilo como tratamiento único durante un corto período en pacientes con resistencia a tratamientos previos, se sugiere su suspensión si los pacientes no responden dentro de las cuatro u ocho semanas. El metrotexato posee potentes efectos inhibidores en la síntesis de citocinas, sin embargo se debe monitorizar por toxicidad hepática, pulmonar y mielosupresión. La evidencia sugiere una efectividad similar a la ciclosporina. La azatriopina posee efectos antiinflamatorios y antiproliferativos, se ha utilizado en pacientes con DA grave con eficacia del 30 al $70 \%$ en la severidad del eccema y el prurito, no obstante, un efecto adverso importante es la mielosupresión $(6,10,20)$.

\section{- Fototerapia}

La fototerapia es un tratamiento de segunda línea considerado en pacientes con lesiones extensas y resistentes a los tratamientos habituales, su uso ha sido aprobado en mayores de 12 años. No se recomienda en exacerbaciones agudas de DA ni tampoco en combinación con ciclosporina oral o con inhibidores de la calcineurina, por riesgo de malignidad. El requisito de asistir al centro hospitalario varias veces por semana para su aplicación limita su uso y una sobredosificación de radiación podría inducir eccema $(2,6,11,17,20)$
- Nuevas líneas de tratamiento farmacológico

- Dupilumab: un anticuerpo monoclonal que inhibe la IL-4 y la IL-13, ha demostrado ser efectivo en pacientes con refractariedad a los corticosteroides tópicos y a la ciclosporina. Entre sus efectos adversos se destacan las reacciones a la inyección local del fármaco y la conjuntivitis. A pesar de que ha supuesto una revolución terapéutica, el alto costo del fármaco y la falta de estudios en niños limitan su uso.

- Tralokinumab y lebrikizumab: son anticuerpos monoclonales dirigidos frente a la IL-13 que han demostrado una mejoría significativa en el SCORAD, el Dermatology Life Quality Index y el prurito $(3,6,21,25)$.

- Tezepelumab: es un anticuerpo monoclonal con acción frente a la vía de la TSLP, esta vía forma parte del eje Th2, fundamental en la interacción entre la epidermis y la activación inmune innata y adaptativa al llevar la respuesta inflamatoria a un fenotipo alérgico, por medio de la activación de las células dendríticas inmaduras que expresan OX40L $(3,12)$. En la actualidad, están activos ensayos clínicos de fase III con fármacos inhibidores de JAK por vía oral. Baricitinib antagoniza a JAK 1 y JAK 2 y está dirigido a pacientes con DA moderada y grave, sin embargo la elevación asintomática de la creatina cinasa 
es uno de los efectos adversos reportados. Upadacitinib y abrocitinib son inhibidores selectivos de JAK $1(3,6)$.

$\mathrm{Se}$ han desarrollado nuevas moléculas de pequeño tamaño que permiten considerar su uso tópico.

- Crisaborol tópico: actúa inhibiendo a la fosfodiesterasa 4 (PDE4) y su uso se ha aprobado en pacientes mayores de 12 años con DA leve y moderada. Su eficacia se ha comparado con los corticosteroides de moderada potencia pero con menor frecuencia de efectos adversos, no se ha evidenciado atrofia cutánea, telangiectasias e hipopigmentación, sin embargo se ha documentado sensación de dolor o quemazón en el lugar de su aplicación. Actualmente se encuentran en investigación los inhibidores tópicos de la vía JAK/STAT. Tofacitinib tópico y ruxolitinib parecen mostrar eficacia en la reducción del EASI $y$ del prurito $(3,6,14,20)$.

\section{- Apoyo psicológico}

El apoyo psicológico se debe considerar en pacientes con desencadenantes emocionales que dificultan un adecuado control de la DA, el estrés emocional conlleva a un aumento del prurito. La frecuencia de problemas psicológicos 0 psiquiátricos en los pacientes con enfermedad moderada o severa es más alta que en pacientes con diabetes mellitus (20).

\section{CONCLUSIÓN}

A pesar de que es una enfermedad crónica y que los pacientes en remisión conservan la predisposición a la DA durante toda su vida, los avances en las nuevas líneas de tratamiento y manejo de la enfermedad son alentadores. Se determinó que en los últimos años, el mayor conocimiento de la patogenia de la DA, más allá del eje Th2, ha condicionado el desarrollo de nuevas terapias sistémicas, entre las que destacan los fármacos biológicos dirigidos frente a la IL-4/13, como dupilumab, tralokinumab y lebrikizumab y los inhibidores de JAK entre los que se incluyen baricitinib, upadacitinib y abrocitinib. A su vez, se han desarrollado nuevas moléculas de pequeño tamaño que permiten considerar su uso tópico, tales como los inhibidores de la PDE4 y de la vía JAK/STAT. Es importante ampliar la investigación de los mecanismos patogénicos, ya que una mayor comprensión del tema podría modificar el curso de la enfermedad e inclusive el desarrollo de otras enfermedades atópicas.

El avance en la investigación de los nuevos tratamientos y la estratificación en los distintos subtipos y subfenotipos de DA parece crucial para llegar a terapias efectivas, a largo plazo y con un perfil de seguridad aceptable. 


\section{REFERENCIAS}

1. Fishbein A, Silverberg J, Wilson E, Ong P. Update on Atopic Dermatitis: Diagnosis, Severity Assessment, and Treatment Selection. The Journal of Allergy and Clinical Immunology: In Practice [Internet]. 2020 [cited 6 April 2020];8(1):91-101. https://doi.org/10.1016/j.jaip.2019.06.044

2. Tanei R. Atopic Dermatitis in Older Adults: A Review of Treatment Options. Drugs \& Aging [Internet]. 2020 [cited 23 March 2020];37(3):149-160. https://doi.org/10.1007/s40266-020-00750-5

3. Munera-Campos M, Carrascosa J. Innovación en dermatitis atópica: de la patogenia a la terapéutica. Actas Dermo-Sifiliográficas [Internet]. 2020 [cited 23 March 2020];111(1):1-17. https://doi.org/10.1016/i.ad.2019.11.002

4. Lara-Corrales I, Bergman J, Landells I, Ramien M, Lansang P. Approach to the Assessment and Management of Pediatric Patients With Atopic Dermatitis: A Consensus Document. Section I: Overview of Pediatric Atopic Dermatitis. Journal of Cutaneous Medicine and Surgery [Internet]. 2019 [cited 9 April 2020];23(5_suppl):3S-11S. https://doi.org/10.1177/1203475419882049

5. Maliyar K, Sibbald C, Pope E, Gary Sibbald R. Diagnosis and Management of Atopic Dermatitis. Advances in Skin \& Wound Care [Internet]. 2018 [cited 11 April 2020];31(12):538-550. https://doi.org/10.1097/01.ASW.0000547414.38888.8d

6. Torres T, Ferreira E, Gonçalo M, Mendes-Bastos P, Selores M, Filipe P. Update on Atopic Dermatitis. Acta Médica Portuguesa [Internet]. 2019 [cited 10 April 2020];32(9):606-613. https://doi.org/10.20344/amp.11963

7. Chu H, Park K, Kim S, Lee J, Park J, Lee K et al. Allergen-specific immunotherapy for patients with atopic dermatitis sensitized to animal dander. Immunity, Inflammation and Disease [Internet]. 2020 [cited 9 April 2020];8(1):1-123. Available from: https://onlinelibrary.wiley.com/doi/full/10.1002/iid3.291

8. Lauritano D, Ronconi G, Caraffa A, Enrica Gallenga C, Kritas S, Di Emidio P et al. New aspect of allergic contact dermatitis, an inflammatory skin disorder mediated by mast cells: Can IL-38 help?. Medical Hypotheses [Internet]. 2020 [cited 9 April 2020];139:109687. https://doi.org/10.1016/j.mehy.2020.109687

9. Fujii M. Current Understanding of Pathophysiological Mechanisms of Atopic Dermatitis: Interactions among Skin Barrier Dysfunction, Immune Abnormalities and Pruritus. Biological and Pharmaceutical Bulletin [Internet]. 2020 [cited 10 April 2020];43(1):12-19. https://doi.org/10.1248/bpb.b19-00088

10. FitzPatrick: Bargot M, Stalder R. Cutaneous lymphoma. In: Wolff K, Goldsmith L, Katz SI. Fitzpatrick's dermatology in general medicine. 9ma ed. Nueva York: McGraw-Hill. 2018. p. 363-381

11. Bekić S, Martinek V, Talapko J, Majnarić L, Vasilj Mihaljević M, Škrlec I. Atopic Dermatitis and Comorbidity. Healthcare [Internet]. 2020 [cited 9 April 2020];8(2):70. https://doi.org/10.3390/healthcare8020070

12. Elsner J, Carlsson M, Stougaard J, Nygaard U, Buchner M, Fölster-Holst R et al. The OX40 Axis is Associated with Both Systemic and Local Involvement in Atopic Dermatitis. Acta Dermato Venereologica [Internet]. 2020 [cited 9 April 2020]; https://doi.org/10.2340/00015555-3452

13. Leonard A, Wang J, Yu L, Liu H, Estrada Y, Greenlees L et al. Atopic Dermatitis Endotypes Based on Allergen Sensitization, Reactivity to Staphylococcus aureus Antigens, and Underlying Systemic Inflammation. The Journal of Allergy and Clinical Immunology: In Practice [Internet]. 2020 [cited 8 April 
2020];8(1):236-247.e3. https://doi.org/10.1016/j.jaip.2019.08.013

14. El Hachem M, Di Mauro G, Rotunno R, Giancristoforo S, De Ranieri C, Carlevaris C et al. Pruritus in pediatric patients with atopic dermatitis: a multidisciplinary approach - summary document from an Italian expert group. Italian Journal of Pediatrics [Internet]. 2020 [cited 9 April 2020];46(1). https://doi.org/10.1186/s13052-020-0777-9

15. Hon K, Lam P, Ng W, Kung J, Cheng N, Lin Z et al. Age, sex, and disease status as determinants of skin hydration and transepidermal water loss among children with and without eczema. Hong Kong Medical Journal [Internet]. 2020 [cited 6 April 2020];26(1):19-26. Available from: https://www.hkmj.org/abstracts/v26n1/19.htm

16. Davidson W, Leung D, Beck L, Berin C, Boguniewicz M, Busse W et al. Report from the National Institute of Allergy and Infectious Diseases workshop on "Atopic dermatitis and the atopic march: Mechanisms and interventions". Journal of Allergy and Clinical Immunology [Internet]. 2019 [cited 8 April 2020];143(3):894913. https://doi.org/10.1016/j.jaci.2019.01.003

17. Katayama I, Aihara M, Ohya $\mathrm{Y}$, Saeki $\mathrm{H}$, Shimojo N, Shoji $S$ et al. Japanese guidelines for atopic dermatitis 2017. Allergology International [Internet]. 2017 [cited 8 April 2020];66(2):230-247. https://doi.org/10.1016/i.alit.2016.12.003

18. A medical emergency in patients with atopic dermatitis: Vera-Kellet $\mathrm{C}$, Hasbún $\mathrm{C}$. Eczema herpeticum: A medical emergency in patients with atopic dermatitis. IDCases [Internet]. 2020 [cited 9 April 2020];19:e00663. https://doi.org/10.1016/j.idcr.2019.e00663

19. Chidwick K, Busingye D, Pollack A, Osman R, Yoo J, Blogg S et al. Prevalence, incidence and management of atopic dermatitis in Australian general practice using routinely collected data from Medicinelnsight. Australasian Journal of Dermatology [Internet]. 2020 [cited 9 April 2020];. https://doi.org/10.1111/ajd.13268

20. Sánchez J, Sánchez M, Macías-Weinmann A, Barreto B, Ensina L, Uriarte-Obando S et al. Systematic review about 10 interventions in dermatitis. A document from the Latin American Society of Allergy, Asthma, and Immunology. Revista Alergia México [Internet]. 2019 [cited 10 April 2020];66(4):426. https://doi.org/10.29262/ram.v66i4.636

21. Ferrucci S, Casazza G, Angileri L, Tavecchio S, Germiniasi F, Berti E et al. Clinical Response and Quality of Life in Patients with Severe Atopic Dermatitis Treated with Dupilumab: A Single-Center Real-Life Experience. Journal of Clinical Medicine [Internet]. 2020 [cited 9 April 2020];9(3):791. https://doi.org/10.3390/jcm9030791

22. Van Zuuren E, Fedorowicz Z, Christensen R, Lavrijsen A, Arents B. Emollients and moisturisers for eczema. Cochrane Database of Systematic Reviews [Internet]. 2017 [cited 8 April 2020];(Issue 2. Art. No.: CD012119.). https://doi.org/10.1002/14651858.CD012119

23. Murota H, Yamaga K, Ono E, Katayama I. Sweat in the pathogenesis of atopic dermatitis. Allergology International [Internet]. 2018 [cited 8 April 2020];67(4):455-459. https://doi.org/10.1016/i.alit.2018.06.003

24. Sugita K, Akdis C. Recent developments and advances in atopic dermatitis and food allergy. Allergology International [Internet]. 2020 [cited 9 April 2020];69(2):204-214. https://doi.org/10.1016/i.alit.2019.08.013

25. Renert-Yuval Y, Guttman-Yassky E. New treatments for atopic dermatitis targeting beyond IL-4/IL-13 cytokines. Annals of Allergy, Asthma \& Immunology [Internet]. 2020 [cited 9 April 2020];124(1):28-35. https://doi.org/10.1016/j.anai.2019.10.005 